\title{
CREATIVIDAD - HERRAMIENTA PARA GESTIÓN DE CRISIS
}

\author{
Vera Cristina Vieira Ribeiro \\ Instituto Politécnico de Coimbra \\ Escola Superior de Educação de Coimbra \\ cristina.veraribeiro@gmail.com
}

Recepción Artículo: 18 mayo 2021

Admisión Evaluación: 18 mayo 2021

Informe Evaluador 1: 29 mayo 2021

Informe Evaluador 2: 02 junio 2021

Aprobación Publicación: 03 junio 2021

\section{RESUMEN}

Sabemos que al contrario que otros animales, el ser humano tiene capacidades genéticas, intelectuales y mentales capaces de crear soluciones que van más allá de la supervivencia, por ello, en este estudio el enfoque consistirá en profundizar el proceso creativo, buscar los orígenes que dan lugar a la materialización de diferentes formas y resultados. Varios estudios nos hacen reflexionar sobre este lugar donde surgen las ideas en el ser humano, algunos apuntan al carácter inconsciente de la mente humana, otros hacia una fuente creativa accesible y disponible para el individuo. Más que reflexionar sobre los condicionantes externos, bien sean sociales, educativos económicos o raciales, el enfoque principal de este artículo es ir en busca del registro o lugar interno, que permite al ser humano conectarse con el estado creativo. Como recurso innato en el ser humano, la creatividad debe ser entendida, analizada y practicada para que pueda ser utilizada en beneficio de la sociedad y la evolución de la vida sobre todo en momentos que las crisis se presentan, bien sea a nivel individual o social.

Palabras clave: creatividad; inteligencia; mente; inconsciente; imaginación

\section{ABSTRACT}

Creativity - a tool for crisis management. We know that unlike other animals, the human being has genetic, intellectual, and mental capacities capable of creating solutions that go beyond survival, therefore, in this study the focus will be on deepening the creative process, looking for the origins that give rise to the materialisation of different forms and results. Several studies make us reflect on this place where ideas arise in the human being, some point to the unconscious character of the human mind, others to a creative source accessible and available to the individual. Rather than reflecting on the external conditioning factors, be they social, educational, economic, or racial, the focus of this article is to look for the internal register or place, which allows the human being to connect with the creative state. As an innate human resource, creativity must be understood, analysed, 


\section{CREATIVIDAD - HERRAMIENTA PARA GESTIÓN DE CRISIS}

and practised so that it can be used for the benefit of society and the evolution of life, especially in times of crisis, whether at the individual or societal level.

Keywords: creativity; intelligence; mind; unconscious; imagination

\section{INTRODUCCIÓN}

La creatividad es un fenómeno atribuido al ser humano, porque es hasta el momento el ser que la utiliza de forma más frecuente y en un gran porcentaje cuando comparada con los demás seres. Es a su vez un concepto multidimensional ya que se relaciona directamente con "aspectos cognitivos, ambientales y de personalidad". Los primeros se relacionan con el conocimiento e la inteligencia, los ambientales se refieren a la educación, al lugar o cultura donde la persona de desarrolla. Los aspectos de personalidad tienen que ver con las características innatas de cada persona, que incluyen la motivación propia y autoconfianza. (Pocinho \& Garcês, 2018) Cuando todas estas características del ser humano están favorecidas, estará también la creatividad, Io que acompaña la capacidad que la persona tiene para responder de manera más capaz a los episodios de crisis, ya sean personales, colectivas o mundiales. Esto se refuerza cuando analizamos situaciones concretas de personas que ante una crisis han conseguido sacar adelante proyectos, cambios em padrones de vida, 0 dones que hasta ese momento no se habían hecho consciente en la realidad. (Los estudios psicológicos demuestran que las personas psíquicamente maduras evolucionan tanto de forma instintiva e impulsiva como racional y lógica. Así, las técnicas que favorecen el autoconocimiento pueden aumentar la creatividad y presentar al individuo la comprensión de que el mundo puede transformarse y permitir la lucha contra lo convencional y lo preestablecido) (Pocinho \& Garcês, 2018, p.55)

\section{METODOLOGÍA Y/O INSTRUMENTOS UTILIZADOS}

Para reflexionar sobre la fuente u origen del proceso creativo en este artículo, se han analizado y señalado varios artículos y publicaciones de diferentes autores donde el principal objetivo es encontrar una lógica y explicación científica para el proceso que ocurre en la mente humana y que genera la creación de formas creativas. Se ha elegido, principalmente, la consulta de revistas de impacto científico para el tema que se pretende investigar, con el fin de garantizar el rigor de los resultados que indicamos en este estudio, sin embargo, solamente las que están reflejadas en las referencias se han tenido en cuenta.

A través de las ideas y/o pensamientos es posible materializar la creatividad, pues de esta forma se hacen visibles y reales los diferentes tipos de invenciones, obras o desarrollos humanos. Antes de que algo exista, surge un pensamiento que se ha convertido en algo real, aunque sea un concepto nuevo.

En este estudio buscaremos, a través de varias investigaciones, el lugar donde surgen las ideas generadas, que dan como resultado el proceso creativo. Por ello, es muy importante tener en cuenta la veracidad de los planteamientos sobre la creatividad porque es un tema transversal que abarca distintas áreas del conocimiento, como veremos en el desarrollo del artículo. Además, este tema exige una adaptación seria y abierta que permita explorar y llegar a los hechos científicos. Las referencias consultadas son heterogéneas y se enfocan tanto en análisis clásicos, así como en otros más contemporáneos. De esta manera, se posibilita una contextualización evolutiva sobre este tema.

\section{DISCUSIÓN}

El fenómeno creativo ha ganado en los últimos años un gran enfoque, sobre todo debido a las sociedades modernas que son extremadamente sugestivas para la creación y la innovación a todos los niveles de la sociedad. Incluso, podemos afirmar que la tendencia será seguir creciendo, ya que el reto social está cada vez más enfocado en el desarrollo, en la creación de las nuevas formas de tecnologías, relaciones, comunicaciones, tendencias y valores. La modernidad ha cambiado la manera en la que comprendemos el mundo y la forma en la que nos relacionamos con él, la libertad que el individuo comparte hoy en día le permite el desarrollo creativo, de esta manera caminamos, seguramente, hacia la era de la creatividad. (Oliveira, 2010) Hemos vivido en los tiempos más 
recientes cambios drásticos, debido a una pandemia mundial, en varios niveles de nuestras vidas, bien sea en las relaciones personales, económicas, sociales, de salud, y seguramente en muchas otras.

Como podremos reflexionar en profundidad un poco más adelante, el ser humano se distingue de otros animales debido, entre otras, a sus capacidades neuronales y mentales, por ello, la creatividad se ha tratado de explicar a lo largo de los siglos. En tiempos ancestrales, los dioses, los seres extra físicos o no físicos eran apuntados e invocados por las religiones o el misticismo para explicar el fenómeno creativo. Por otra parte, las teorías filosóficas que apuntaban hacia el individuo estaban inspiradas en algo divino para la creación y para practicas creativas, y estas creencias siguen estando de manera inconsciente, y algunas de forma consciente, aún presentes en los valores de la humanidad. Por ejemplo, Platón apuntaba que "ser el artista, en el momento de la creación, era ser un agente de un poder superior, perdiendo el control de sí mismo." (Oliveira, 2010) Esta afirmación indica un proceso incontrolable que ocurre en la mente humana y que posibilita el surgimiento de creaciones, sin que el individuo las esté buscando e intentando crear objetivamente. El marco en el que floreció la cuestión sobre la creatividad fue el periodo del Renacimiento, en que la creatividad ganó un gran impulso teniendo en cuenta todo el desarrollo y evolución artística de la época.

Es en este momento en el que, de forma universal, la creatividad gana un estatus que le hace posicionarse como una característica innata en el ser humano y que puede ser desarrollada. Es sobre todo en el área de la psicología que los estudios sobre este tema traen nuevas e importantes intervenciones, las investigaciones empiezan a apuntar hacia el estudio de la creatividad de una forma más objetiva y racional que poco a poco se ha introducido en el conocimiento general en la sociedad.

Apuntamos algunos nombres e ideas sobre el concepto de creatividad gracias a autores que han reflexionado sobre este tema, como por ejemplo, Ellis Paul Torrance (1915-2003), psicólogo americano e investigador reconocido por estudiar la creatividad, presentó la idea de que la creatividad "es el proceso de hacerse sensible a crear respuestas, soluciones para algo que está en falta, es crear nuevas hipótesis, comprobar si son útiles y volver a recrearlas, finalmente, compartiéndolas y compartiendo el resultado. Por otro lado, Louis Leon Thurstone (18871955), investigador del área de la psicométrica y psicofísica añade a esta definición que el resultado de lo que se ha creado como fruto del uso de la creatividad, tiene que ser necesariamente algo nuevo. Por su parte, Matisse, un conocido artista francés, compartía que la creatividad era algo subjetivo, es decir, expresar algo que está en el interior de cada ser humano y que, a su vez, se puede potenciar con la ayuda de elementos del mundo exterior, para así alimentar este sentimiento de creación para alimentar a su vez este sentimiento de creación que se puede potenciar con la ayuda de elementos del mundo exterior. (Oliveira, 2010) Las investigaciones más recientes nos indican que la creatividad apunta para una característica humana que es especialista en la resolución de problemas que afectan al ser humano, que pueden ir desde situaciones prácticas del día a día, a formas de relacionarse, innovaciones científicas, obras literarias, artísticas, en definitiva, es una característica que busca la evolución constante.

Es interesante observar el carácter poco lógico y racional de la creatividad. "Podemos considerar la creatividad como "una fuerza", algo que tenemos que escuchar, entender y dominar, para que así podamos beneficiarnos de la totalidad de su potencial." (Oliveira, 2010) La creatividad es una característica humana que no ha sido tan profundizada a lo largo del tiempo, sin embargo, es el indicador que está por detrás de todas las invenciones y comodidades que tenemos en nuestra vida actual. Merece, por ello, la inversión y la investigación profunda para definirla, encontrarla y desarrollarla, ya que está en este concepto el potencial humano, además, es también a través de la creatividad y, después del salto evolutivo que ha tenido la humanidad, a lo largo de los años, la principal característica que distingue el hombre de otros animales.

Encontrar esta fuerza, este lugar interno que permite al individuo entrar o reconocer el proceso creativo sigue siendo un gran misterio, sólo comparable a la complejidad de la creación de la vida en todos sus componentes, que van desde el aire en este planeta hasta la formación de diferentes universos, todo con una organización claramente matemática. Sabemos que esta particularidad es innata al hombre y que algunas personas tienen más 


\section{CREATIVIDAD - HERRAMIENTA PARA GESTIÓN DE CRISIS}

facilidad en encontrarla y de desarrollarla que otras. Varios condicionantes pueden facilitar o bloquear el acceso a este estado de fluidez mental que da lugar a que nazcan nuevas y novedosas creaciones.

Profundizando un poco más en el origen y las características mentales que distinguen al hombre de otros animales, sabemos que la curiosidad es una de las capacidades que ha permitido la evolución y el desenvolvimiento de la especie. Cuando nos referimos a curiosidad, y damos lugar y espacio para que ella sea desarrolle, surgen de inmediato en el campo mental una gran cantidad de preguntas y la búsqueda de sus respuestas atrae a la motivación, que fomenta la creatividad, incluso, por ejemplo, en lo que se refiere a investigación científica, pues es una forma de creatividad en su esencia y que encuentra en la lógica una forma de expresión. La curiosidad se convierte en un recurso utilizado por la creatividad para generar productos y producciones, tengan éstas el carácter que tengan. En este punto enfocamos la creatividad desde un lugar objetivo y definible, cuando en un individuo despierta una pregunta que hasta el momento no tenía respuesta, utiliza como recurso la creatividad para encontrar nuevas formas y respuestas posibles que contesten a su objeción.

Otro aspecto muy relacionado con la creatividad y que es relevante señalar, además de que podría estar relacionado con la inteligencia, es la fantasía. Cuando investigamos sobre la noción de fantasía, las indicaciones descubrimos que se trata de algo creado or la mente consciente. Es la fantasía la que trae al individuo la conceptualización de lo que es imaginado, es decir, no es real, por lo que no aporta importancia y significado a las creaciones. Para comprender e identificar mejor el concepto de fantasía, debemos adentrarnos en el inconsciente del ser humano y, como dijo Freud, uno de los mejores investigadores, el hombre feliz no tiene fantasías, sino que las fantasías son deseos no realizados. Esto nos lleva a una idea muy clara, la fantasía es algo creado por la mente inconsciente, Jung considera que la fantasía es la corrección de la realidad que no se ha realizado, pero se imagina como algo realizado, aunque sólo sea imaginado. (Comparato, 2004) Parece importante señalar que la fantasía como fenómeno no está directamente relacionada con la creatividad o con la creación de ideas que aportan algo novedoso y único, sino que se trata más de un condicionamiento mental que se desarrolla en el ser humano, pero no tiene un carácter evolutivo, más bien condicionador del comportamiento.

\section{Posible acercamiento a la fuente del potencial creativo}

Por lo general, y desde tiempos inmemoriales, el ser humano quiere saber el cómo, el porqué, el cuándo y el dónde, esta característica es la base de la creación, de la creatividad. Comparto, (2004) refiere que "a semejanza del Creador, el humano también es creador y creativo". Si miramos la diversidad en este planeta verificamos que la creación es sin duda extremadamente creativa, tenemos una diversidad casi infinita de seres, especies, colores y formas. Y si miramos fuera de nuestro planeta, lo mismo se repite, por ello, es fácil concluir que el Creador de la vida es extremadamente creativo.

Tal vez sea importante en este momento comprender el significado de creador que aquí usamos para este estudio, ya que el origen etimológico de la palabra es el mismo que el de creatividad. Creador y creatividad tienen la misma raíz, del latín creare, que significa capacidad de crear, producir o inventar cosas o nuevos conceptos. (Oliveira, 2010) Puntuamos este hecho como forma de reforzar lo que lo que explicaremos a continuación.

Estudios recientes, junto con otros no tan recientes pero que ya apuntaban hacia lo que describimos a continuación, confirman que los físicos han descubierto que en escalas minúsculas las partículas con las que se forman nuestros cuerpos son las mismas que las partículas de las estrellas o de otros planetas. Esto nos dirige a la conclusión de que, tanto de la realidad física, como al nivel de las partículas, todo está conectado y es infinito. (Braden, 2020). Añadimos a este hecho el concepto de los ciclos naturales y biológicos que encontramos en la naturaleza y para los cuáles no tenemos ninguna explicación lógica u objetiva, no sabemos quién los controla, y lo mismo ocurre con el ritmo biológico del cuerpo humano, simplemente ocurre, no es elegido por el individuo. Al igual que podríamos decir del movimiento de los planetas o de la creación de los universos, tanta diversidad y cantidad, pero que, a pesar de los avances, todavía seguimos sin conseguir explicar esta fuerza que mantiene todo y todas las formas de vida a un ritmo matemático, sincrónico y organizado. Es a este lugar al que nos lleva 
la reflexión sobre la creatividad, lugar que no es físico y del que surgen, brotan las ideas o pensamientos que cuando se analizan y ejecutan por la mente, ésta los convierte en cosas o conceptos físicos, bien sea un libro, un nuevo producto, 0 una invención tecnológica. En sus pesquisas Gregg Braden (2020), investigador y especialista en sistemas informáticos, llama a este fenómeno la matriz divina, dando nombre a lo que podríamos definir como la fuente, el origen de donde nacen las ideas creativas; la matriz o el campo desconocido, pero a la vez lleno de información y capacidad donde todas las cosas visibles e invisibles que conocemos existen. Braden (2020) indicó tres formas simples de definirla: 1) receptáculo en el interior del cual existe todo; 2) los puentes entre nuestros mundo interiores y exteriores; y 3) el espejo que refleja todos los días nuestros pensamientos, sentimientos, emociones o creencias. Este lugar de difícil conceptualización que, al contrario de una emisión de televisión o de radio que es creada en un determinado lugar y recibida en otro, este campo se produce en todo lugar y todo el tiempo. Varios estudios en el área de la física y la física cuántica señalan que este campo surge a raíz de lo que frecuentemente llamamos el big bang, es decir, la liberación que se produjo en ese momento ha permitido la expansión de esa energía que se expande sin parar. Otro factor muy interesante que debemos tener en cuenta es que este lugar es dotado de una inteligencia matemática, como hemos comprobado con argumentos en el epígrafe. Cuando analizamos el funcionamiento harmonioso y sincrónico del movimiento de los planetas que permite que no choquen, la capacidad del cuerpo humano para funcionar o sanarse, el ciclo de la naturaleza y su abundancia de alimentos, o bien el ciclo de las mareas que se conecta con los ciclos de la luna. Tanto podríamos aportar y seguramente se estudiará sobre este tema en los próximos años, es un territorio que no obedece a la lógica tradicional de los parámetros o metodologías actuales de la ciencia. Sin embargo, la curiosidad humana no tiente límites y seguramente llegará un momento en el que las creencias fuertemente enraizadas en las mentes colectivas darán lugar y apertura a nuevas conceptualizaciones, más contemplativas y ampliadas.

Un ejercicio perfecto que nos permite acceder a este campo de donde nacen las ideas creativas es cerrar los ojos por unos segundos, relajarse, respirar hondo, dejar que surjan imágenes libremente en nuestra mente y observarlas con atención, detalladamente, escoger y analizar una de ellas y, finalmente, elegir una palabra para describirla y escribirla en un papel, se podrá repetir este proceso varias veces. En este momento, el individuo que haga este ejercicio estará perfectamente conectado a ese lugar o campo creativo de donde surgen las ideas y donde se desarrolla todo el proceso creativo. (Comparto, 2004)

Analizamos también varios estudios que apuntan hacia la facilidad que tienen algunas personas y que reciben el nombre de insight y que de forma involuntaria traen la respuesta a una curiosidad o desafío. Estas informaciones surgen esencialmente cuando las personas están relajadas, en los momentos que anteceden al sueño, o cuando están en la ducha. La explicación se encuentra en que en estos momentos de relajación la mente humana emite un estado de ondas Alpha que los estudios reconocen como el estado en el que el proceso creativo se desarrolla con más fluidez y abundancia. "Las ondas cerebrales se refieren a la actividad eléctrica producida por el cerebro, se clasifican según letras griegas como: delta, theta, alpha, beta y gamma en dependencia del rango de Hertz (Hz) en el cual se producen." (Morales, 2017)

La creatividad es la realización de las potencialidades del ser humano, es su genialidad, su carácter innovador en acción, por eso el individuo debe tener en cuenta su apertura a las nuevas experiencias, opiniones, hipótesis, así como tener en cuenta si está abierto a probar o innovar, además de si se permite estar plenamente presente en cada momento, permitiendo de esta forma realizar una conexión más fuerte a un estado de tranquilidad que le va a permitir acceder al estado creativo. (Brito et al., 2009)

\section{Inteligencia y creatividad}

Todo lo que hemos explorado hasta este momento sobre la creatividad nos indica que más que una habilidad, es una capacidad que está a la disposición del ser humano y que le permite ser y estar en un campo de múltiples e infinitas posibilidades. La creatividad es dinámica y emprendedora, un proceso que puede ser provocado o dirigido, puede ser una elección por parte del individuo o puede ocurrir involuntariamente, puede estar contextuali- 


\section{CREATIVIDAD - HERRAMIENTA PARA GESTIÓN DE CRISIS}

zado en situaciones profesionales, pero incorpora un sinfín de aplicaciones. Seguramente, a cualquiera le ha tocado esta experiencia, en algún momento de la vida, le surgió un desafío y en algún momento la solución llega a su mente a través de un pensamiento como si de magia se tratara. En otros momentos, se trata de un proceso que se elige poner en marcha, que lleva en sí su origen y su meta, se puede decir que "abarca aptitudes, fuerzas y talentos que se manifiestan con diferentes grados de calidad e intensidad, en distintas circunstancias y contextos de la vida." (Hernández et al., 2015). Los exámenes que permiten medir el grado de inteligencia están bastante popularizados y son bastante conocidos, sin embargo, queda por comprender como se relacionan estos dos componentes del funcionamiento de la mente humana. Sabemos que las creaciones no siempre se relacionan con el grado de inteligencia del individuo, pero esta última capacidad puede resultar importante cuando hay que desarrollar la idea durante un proceso creativo, aunque las dos pueden resultar más o menos evidentes en determinados individuos.

Es importante enfocar la inteligencia humana como soporte para que la creatividad se pueda desenvolver. Estudios sobre psicología de la inteligencia conceptúan la inteligencia como una construcción del sujeto que añade nuevas dimensiones a los objetos externos. (Alves \& Castro, 2015). Esto indica que el ser humano estructura la realidad externa según sus propias interpretaciones, creencias o patrones mentales, donde una perspectiva individual a todo lo que se presenta. Cuando nos adentramos en el concepto de inteligencia con facilidad, comprendemos la apertura mental necesaria para que se permita la fluidez por donde la creatividad se hace consciente.

La creatividad supone más que la aparición de ideas o pensamientos, lo que realmente define la creatividad es la creación de algo nuevo, que no se ha hecho antes y esto le confiere un carácter de genialidad, tiene un carácter innovador y apropiado. Podríamos añadir que creatividad seria como una capacidad que permite al individuo crear nuevos modos y adaptarse en diferentes contextos, reaccionando de una manera original a los retos o desafíos que se presentan. (Brito et al., 2009)

Varias pesquisas científicas han indicado que el intelecto humano es dotado de estructuras muy complejas y por ello varias discusiones se generan sobre todo cuando se relaciona el pensamiento divergente y la producción creativa. Podríamos afirmar que el primer aspecto se trata de un proceso controlado conscientemente por el ser humano, sin embargo, el segundo nos Ileva de nuevo al concepto más abstracto e indefinible que pasa a través del cerebro humano y se hace consciente. Existen diferencias entre pensamiento divergente, que nos remite a la solución de problemas, para los que hace falta que un reto 0 dificultad se presente para solucionarlo. La creatividad se caracteriza por la ausencia de lógica. (Alves \& Castro, 2015). Citando Kneller, (1978, p. 13) Alves y Castro (2015) transcriben "tan flexible y caprichoso fenómeno es la creatividad, que mal podemos definirla". Señalando la increíble descripción que revela la creatividad como proceso mental y emocional que exige de la persona que la quiere desenvolver, entrega y desprendimiento total a valores, conceptos existentes en su consciente. Pero, esta es la respuesta para quienes quieren encontrar la novedad, la originalidad o genialidad de una creación, bien sea tecnológica, literaria, científica u otras. Se podrá señalar cuatro criterios que indican que una idea es resultado de un proceso creativo: 1) si la idea es nueva y útil, bien sea para el individuo o la sociedad; 2) si la idea invalida ideas anteriores, crea evolución; si la idea resulta de importante motivación y persistencia; 3) si la idea ha empezado de un lugar inicialmente vago y finalmente aporta algo. Con estas directrices podemos entender que la realización creativa ultrapasa la relación entre el individuo y el mundo, aunque como vimos anteriormente el conocimiento previo puede tener influencia en la forma en la que se presenta una nueva creación. (Brito et al., 2009)

Es un desafío definir si la creatividad es un fenómeno mental diferente de la inteligencia, lo que sí es unánime en estudios recientes es que todo individuo tiene la capacidad de desarrollar ambas habilidades. Aunque unos acceden al proceso creativo con más facilidad que otros, lo mismo ocurre con la inteligencia, sabemos que algunas personas tienen una propensión más facilitada para solucionar problemas que otras. La inteligencia está más relacionada con la capacidad de raciocinio del individuo, la lógica se puede medir, mientras que la creatividad 
difícilmente se mide. La teoría de la inteligencia desarrollada indica en un estudio que la inteligencia y la creatividad son un complemento y funcionan en equilibrio. respetando la inteligencia interna, que se refiere a conocimientos del pasado en el individuo, la inteligencia creativa que sería la capacidad de crear algo nuevo y novedoso y la inteligencia empírica, que sería la capacidad de adaptación. (Comparato, 2004)

Howard Gardner, psicólogo cognitivo y educacional, presentó la teoría de las inteligencias múltiples indicando que el individuo tiene un conjunto de inteligencias múltiples y que estas pueden ser independientes entre sí. Obviamente, el ser humano puede tener en sus características personales varios tipos de inteligencias, o incluso, cada individuo puede tener diferentes niveles de evolución en de las varias inteligencias que habitan en su existencia. (Oliveira, 2010)

La inteligencia humana puede ser clasificada en diferentes tipos o grupos, lingüística o verbal, lógica o matemática, especial, musical, corporal o cinestésica, interpersonal o intrapersonal, existencial, emocional. En todos ellos es posible la existencia de una función creativa, ya que esta está en el origen y antes de cualquier concepto mental. Desde una perspectiva neuropsicológica cada uno de estos tipos de inteligencia están presentes y son controlados por diferentes regiones cerebrales, o más bien sistemas específicos que controlan o cooperan con la realización de una tarea. (Brito et al., 2009)

Por otra parte, varios estudios sobre la relación entre la creatividad y la inteligencia se han generado desde cinco perspectivas: 1) la creatividad como subconjunto de la inteligencia, 2) la inteligencia como subconjunto de la creatividad, 3) la creatividad y la inteligencia son dos constructos que se superponen, 4) la inteligencia y la creatividad son lo mismo y, por último, 5) la creatividad y la inteligencia no tienen relación, como conjuntos separados. (Morales, 2017) Estos indicadores generan bastante contradicción y abren enormemente todas las posibilidades de relación entre la creatividad y la inteligencia humana.

\section{CONCLUSIONES}

Las principales conclusiones que podemos analizar en este estudio del fenómeno creativo tienen que ver esencialmente con la complejidad del sistema mental humano, en el que la creatividad se incluye, ya que no es cuantitativo y conlleva una dificultad de análisis objetiva. Por eso, las observaciones que se han considerado en este estudio han tenido como base a diferentes estudios e investigaciones no solamente de carácter objetivo, sino también de carácter subjetivo e interpretativo de las referencias utilizadas.

En el intento de responder a la pregunta qué es la creatividad, han surgido algunas ideas y definiciones que nos indican que es un don divino o surge como resplandores de inspiración, asociación entre locura y creatividad. Hoy, teniendo en cuenta investigaciones y pesquisas sabemos que, aunque algunas personas tengan una mayor facilidad para ejercer las habilidades creativas, estas pueden ser desarrolladas y primoreadas con la práctica de ejercicios y técnicas.

Ha sido bastante evidente la referencia hecha por varios autores de que el fenómeno creativo es algo innato e intrínseco al ser humano, es una característica que puede ser desarrollada, está disponible al individuo como potencial latente. Sin embargo, es necesario tomar consciencia, lo que implica una voluntad, una entrega para una parte del ser que no es racional y explicable con un lenguaje matemático o exacto. Ha sido aun así comprender el funcionamiento de ese sistema interno que nos permite desarrollar y traer innovación a la vida más sencilla hasta la más compleja. Hemos profundizado en la creatividad como una fuerza que nos permite dar respuestas que otros animales no pueden, teniendo en cuenta sus condiciones neuronales, desenvolver de forma consciente.

Considerando y profundizando esta condición diferenciadora del ser humano con relación a otros animales, verificamos que efectivamente hay algo que nos diferencia, que somos conscientes de la vida, nos preguntamos y cuestionamos por ella. A la vez que a través de la creatividad buscamos respuestas a cuestiones o problemas que permiten al individuo crecer más y más y evolucionar en lo que se refiere a situaciones prácticas o complejas. Sin embargo, sabemos que factores externos pueden condicionar fuertemente el desenvolvimiento de esta característica en los individuos. Hablamos de condiciones físicas, económicas, educacionales, financieras, geográfi- 


\section{CREATIVIDAD - HERRAMIENTA PARA GESTIÓN DE CRISIS}

cas, culturales que pueden sin duda provocar una disminución en la capacidad creativa de un ser. Fácilmente se comprende que una persona que está preocupada por su necesidad fisiológica de alimentación o de seguridad no puede estar con su creatividad tan desarrollada como la que está plenamente nutrida y segura.

Verificar y comprender la relación entre la creatividad y la inteligencia humana nos abre más puertas a la exploración y a la investigación. Hemos explicado que las dos particularidades se complementan, podrán estar más o menos perfeccionadas individualmente en cada individuo, pero el potencial está disponible. Lo mismo ocurre con el hecho de que los tipos de inteligencia puedan estar más o menos despiertos en cada individuo. Lo que diferencia la creatividad es que esta parece existir ya antes de que la inteligencia intervenga, es decir, la creatividad es una puerta abierta y es la inteligencia la que organiza y da forma a las ideas que surgen a través de los pensamientos.

La inteligencia en el ser humano se relaciona directamente con la creatividad, sin embargo, lo que no es claro si son la misma competencia o pertenece a funciones diferentes en la mente. Sin embargo, en este estudio, enfocamos sobre todo la inteligencia humana como soporte para que la creatividad se pueda desenvolver. Estudios sobre psicología de la inteligencia conceptúan la inteligencia como una construcción del sujeto que añade nuevas dimensiones a los objetos externos. Esto indica que el ser humano estructura la realidad externa según sus propias interpretaciones, creencias o patrones mentales, donde hay una perspectiva individual en todo lo que se presenta. Pero, cuando nos adentramos en las definiciones de inteligencia la comprendemos más como estructura en la mente humana que permite que la innovación se manifieste, creando una apertura mental para que se permita la fluidez por donde la creatividad se permita hacer consciente.

Es interesante e importante seguir investigando, buscando información, que los investigadores se abran a nuevos conceptos en la ciencia y en la investigación. Urge que sea posible complementar y añadir a los estudios Ios fenómenos que están en un campo subjetivo del conocimiento, pero que es tan válido como el racional u objetivo. La creatividad es algo que no se puede cuantificar, es una característica interna que existe y es parte del ser humano, sin embargo, de difícil definición en los parámetros de la ciencia.

\section{REFERENCIAS BIBLIOGRÁFICAS}

Alves, M. \& Castro, P. (2015). Criatividade: histórico, definições e avaliação. Revista Educação, vol 10, n²-2. http://revistas.ung.br/index.php/educacao/article/view/2161/1611

Braden G. (2020). A matrix divina, lua de papel.

Brito, R. F. Vanzin T. \& Ulbricht, V. (noviembre, 2009). Reflexões sobre o conceito de criatividade: sua relação com a biologia do conhecer.

http://pepsic.bvsalud.org/scielo.php?script=sci_arttext\&pid=S1806-58212009000300017

Comparato, D. (2004). Da Criação ao Guião. A Arte e a Técnica de Escrever para Cinema e Televisão. Cascais: Pergaminho.

Hernández Arteaga, I.; Alvarado Pérez, J. C. \& Luna, S. M. (2015). Creatividad e innovación: competencias genéricas o transversales en la formación profesional. Revista Virtual Universidad Católica del Norte, 44, 135151. http://revistavirtual.ucn.edu.co/index.php/RevistaUCN/article/view/620/1155

Morales, C. (agosto, 2017) La creatividad, una revisión científica. Arquitectura y Urbanismo, vol. XXXVIII, núm. 2, mayo-agosto, 2017, pp. 53-62

https://biblat.unam.mx/pt/revista/au-arquitectura-y-urbanismo/articulo/la-creatividad-una-revision-cientifica

Oliveira, J.M. (maio, 2010). A Criatividade - uma Abordagem Transversal à Origem, aos Processos, Técnicas, Efeitos e Experiências, Universidade do Minho

https://repositorium.sdum.uminho.pt/bitstream/1822/44728/1/Jos\%C3\%A9\%20Miguel\%20da\% 20Silva\%200liveira.pdf

Oliveira, Z. M. (2010). Fatores influentes no desenvolvimento do potencial criativo, https://www.scielo.br/pdf/estpsi/v27n1/v27n1a10.pdf 
Pocinho, M. \& Garcês S. (2018) Psicologia da Criatividade, Universidade da Madeira https://digituma.uma.pt/bitstream/10400.13/2006/1/Psicologia\%20da\%20Criatividade.pdf

Nakano, T. (2018). A criatividade pode ser medida? Reflexões sobre métodos utilizados e questões envolvidas. Arquivos brasileiros de Psicologia.

http://pepsic.bvsalud.org/scielo.php?script=sci_arttext\&pid=\$1809-52672018000100010

Nakano, T. (2020). Métodos Atuais para Avaliação da Criatividade: Vantagens e Questionamentos. Aval. psicol. vol.19, n.1, pp. 97-105.

http://pepsic.bvsalud.org/scielo.php?script=sci_arttext\&pid=\$1677-04712020000100012

Oliveira, Z. (2010). Alguns instrumentos para se medir a criatividade. Avaliação Psicológica, 9(3), 495-497.

http://pepsic.bvsalud.org/scielo.php?script=sci_arttext\&pid=\$1677-04712010000300016 
